\title{
Analysis of Quencher Diffusion in Chemically Amplified Resists and Its Effect on Imaging Characteristics
}

\author{
Keiko Hattori, Shoji Hotta*, Takuya Hagiwara, Hiroshi Fukuda
}

Central Research Lab., Hitachi Ltd., Kokubunji, Tokyo 185-8601, Japan

*Device Development Center, Hitachi Ltd., Ohme, Tokyo 198-8512, Japan

\begin{abstract}
Diffusion of quencher contained in most chemically amplified resists today can cause proximity effects such as dependence of pattern feature size on pattern density or iso-dense bias. Diffusion coefficients were measured for several quencher compounds, and significantly large diffusion coefficients (e.g. $0.003 \mu \mathrm{m}^{2} / \mathrm{sec}$ at $100{ }^{\circ} \mathrm{C}$ ) were observed compared with those observed for acid. To evaluate the influence of quencher diffusion on macroscopic imaging behavior of resists, a new resist model for mutual diffusion of acid and quencher was introduced and was implemented to our in-house lithography simulator. Simulation results based on the model explained various proximity effects observed on real wafers, assuming quencher diffusion length as large as $0.2 \mu \mathrm{m}$. This is significantly long compared with acid diffusion lengths reported and agrees with the results of direct measurement. Our results suggest that very small amount of quencher can have a strong influence on imaging characteristics of chemically amplified resists.
\end{abstract}

Keywords: chemically amplified resist, acid, quencher, diffusion, simulation, proximity effect.

\section{Introduction}

As circuit geometry size becomes smaller than the exposure wavelength of the light used in patterning LSIs, proximity effects increase such as pattern size dependence on pattern density, making it difficult to keep the fidelity of final (etched) pattern features to the original designs. It has been shown that this strongly depends on resist materials and processes and cannot be explained by the optical effect only. To clarify this phenomenon, several factors such as acid diffusion in chemically amplified resist and microloading effect in etching have been investigated. For practical simulation purpose, on the other hand, semi-empirical models have been introduced to describe these complicated resist process behaviors. At this moment, however, there is no single physical model which can fully describe complex behavior of today's state-of-the-art chemically amplified resist processes.

Most chemically amplified resists today contain quencher materials for suppressing resist instability such as the post-exposure delay (PED) effect and T-top cross-sectional profiles. Also, quencher is expected to improve critical dimension (CD) controllability by compensating for the acid diffusion in unexposed region because it quenches photo-generated acid diffused into unexposed region.

In this paper, we focus our attention on the behavior of quencher in chemically amplified resist films. Though several types of materials have been used as quencher materials $[1,2]$, most of them are usually small molecule compounds. Such small molecules are expected to easily move in the resist films, and this may affect patterning characteristics. For example, while quencher in the exposed region is consumed in quenching photo-generated acid, quencher in the unexposed region diffuses to the exposed region. If the diffusion length of quencher is long enough, the amount of quencher provided from unexposed region to exposed region depends on the area of unexposed region, resulting in the pattern density dependence of final resist feature size as illustrated in Fig. 1. 


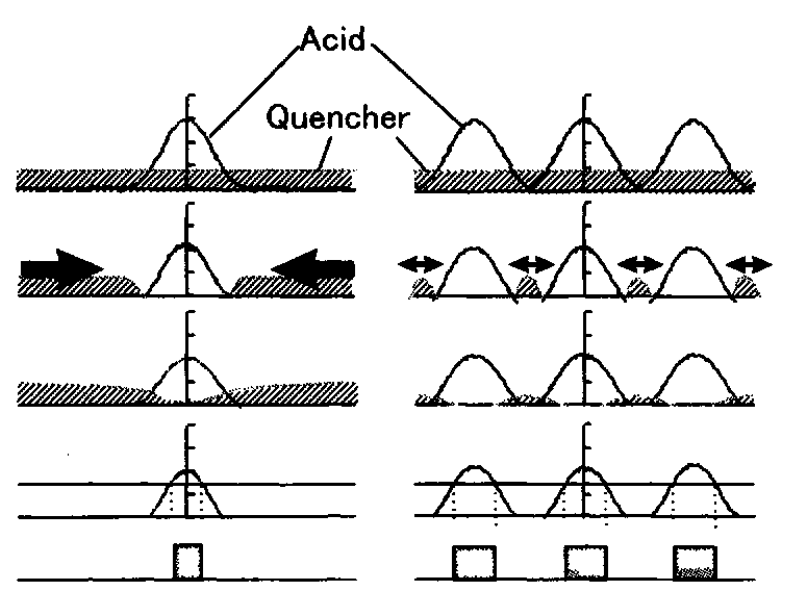

Fig 1 Influence of quencher diffusion in isolated and dense patterns.

This paper discusses the influence of quencher diffusion on macroscopic behavior of resist materials such proximity effects. We expect that this provides a better model for fast pattern geometry simulation, resulting in a higher accuracy in OPC verifications and OPC rule generations. Also, we expect that better understanding of relation between the material composition and its macroscopic behavior is useful for controlling resist material itself. In the following sections, we discuss (1) measurement of quencher diffusion coefficient in resist films, (2) a new resist model including mutual diffusion of acid and quencher and quenching reaction, and (3) comparison between simulated and experimental results to show the validity of the model.

\section{Measurement of quencher diffusion range}

\subsection{Experimental procedure}

Many researchers have measured the diffusion length of acid using various methods. For measuring the quencher diffusion length, we modified the method introduced by Schlegel, et. al. [3]. The experimental procedure is as below and is illustrated in Fig. 2.

An negative tone chemically amplified resist film with $2-\mu \mathrm{m}$ thickness was coated on a quartz substrate as a quencher diffusion detector (detector film). As a diffusion source, the same negative tone chemically amplified resist $(0.8-\mu \mathrm{m}$ thickness) containing quencher was coated on a $\mathrm{Si}$ substrate covered with $\mathrm{Al}$ foil on top. The two resist films were pre-baked at $100{ }^{\circ} \mathrm{C}$ for $120 \mathrm{sec}$. Then the diffusion source film was transferred to the surface of diffusion detector film mechanically, resulting in the sharp boundary between quencher containing region and quencher free region (Fig. 2a). Subsequent thermal treatment (diffusion baking) promoted quencher diffusion into the detector film from the source film (Fig. 2b). After the diffusion baking, i-line light was irradiated from backside of quartz. substrate to generate acid in the detector film. Because the resist was highly transparent at i-line wavelength, acid was generated uniformly in the film (Fig. 2c). The quencher diffusing into the detector film could quench photo-generated acid. Then post exposure baking promoted cross-linking reactions in the region where acid remains (Fig. 2 d). To minimize the influence of PEB on the quencher diffusion, weak condition was used for PEB $\left(70{ }^{\circ} \mathrm{C}, 120 \mathrm{sec}\right)$. Subsequent development with TMAH (2.38\%) removed soluble region where diffused quencher deactivated acid catalyst (Fig. 2e). Then hole depth was measured with a Tencor Alphastep 200 and referred as diffusion range in this article.

Negative type detector film is composed of Novolak resin (100), 1,3,5-tris (2-(2hydoroxypropyl))benzene (Triol-3) (20) and Diphenyliodonium trifulate (DPIOTf) (2.15) (wt. Ratio)[4]. As quencher compounds, 2Benzylpyridine (BP), 2,2'-dipyridyl (DP), 4,4'Diphenyl-2,2'-dipyridyl (DPhDP), 1,3-Di(4pyridyl)propane (DPP), and 4methylaminopyridine (AP) were evaluated[5].

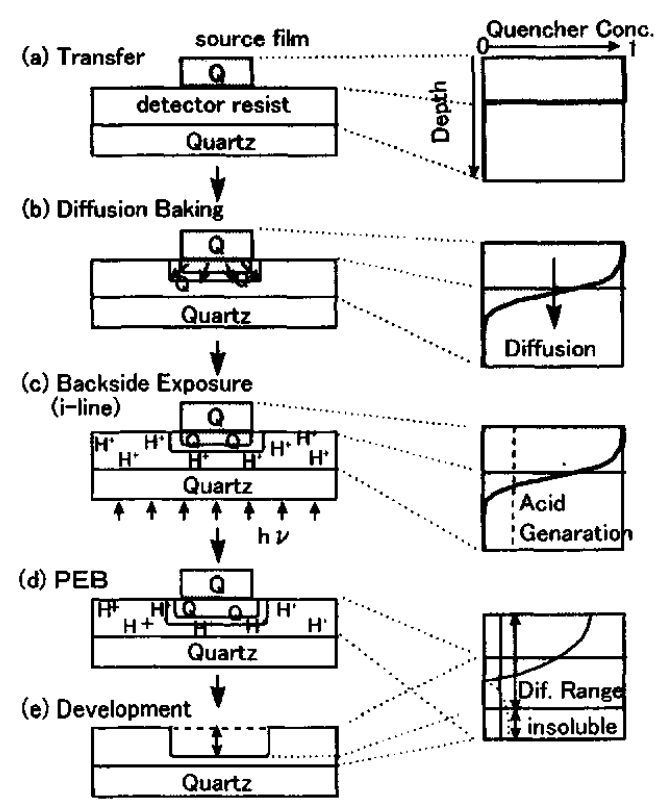

Fig. 2 Procedure of quencher diffusion range measurement. 
The initial quencher concentration in the source film was set equal to the initial photo acid generator (PAG) molar concentration.

\subsection{Result}

Diffusion time dependence of the diffusion range was measured for several diffusion temperatures and quencher compounds. The results for 2,2'-dipyridyl (DP) are shown in Fig. 3. It is shown that the diffusion has the Fickean nature. The other quenchers also showed a similar behavior. The results indicate that diffusion range of typical quencher compound is well above a half micron under conventional PEB conditions.

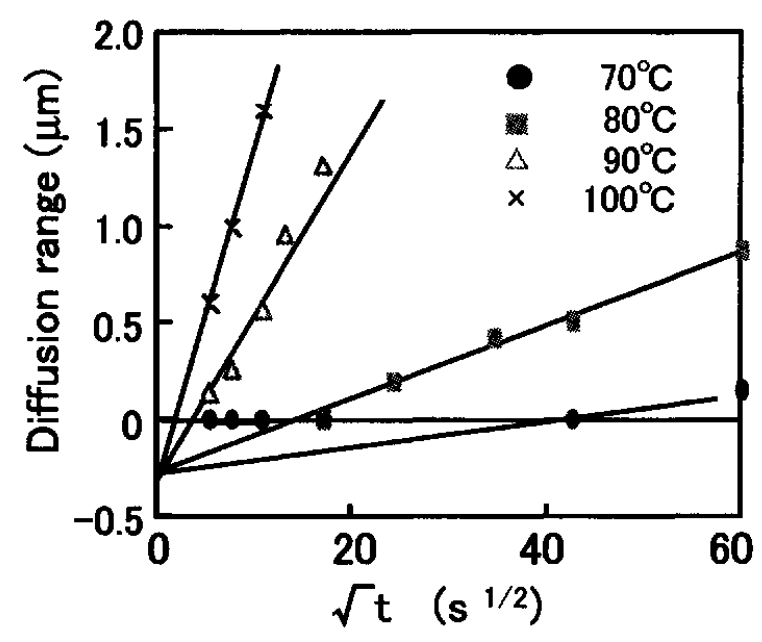

Fig.3 Time dependence of dissolution depth under various diffusion temperature.

Each curve in Fig. 3 doesn't cross the origin, which can be attributed to several reasons as below. (1) The negative tone resist material used. here has some film thickness loss. (2) Some induction time may be required for quartz substrate to reach the target temperature due to its low thermal conductivity, resulting in some offset in PEB time.

Irradiation dose (=the amount of photo generated acid) dependence of the diffusion range was also measured, and the result is shown in Fig. 4. Diffusion baking was performed at $100{ }^{\circ} \mathrm{C}$ for 300 sec. After diffusion, the quencher concentration in the detector film is expected to decrease along the depth direction. Because the amount of acid required for making the film insoluble increases with the quencher concentration, this plot is expected to directly

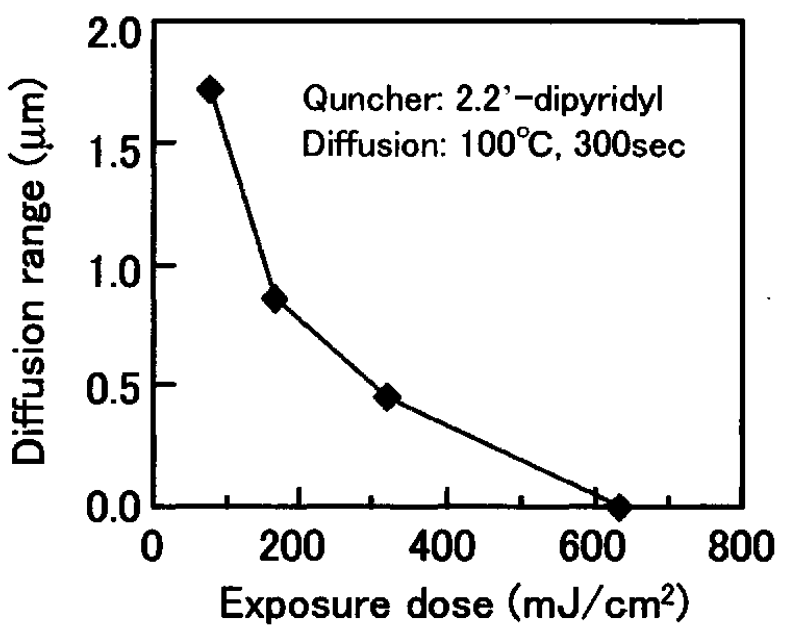

Fig. 4 Diffusion range dependence on i-line flood exposure dose.

reflect the depth profile of quencher diffused into the detector film.

In the next section, we will estimate the diffusion coefficient of the quencher from these results.

\section{Estimation of quencher diffusion coefficient}

We used following two methods to identify the diffusion coefficient of quencher in resist film. All the concentration is normalized by the initial concentration of PAG in the detector and the source films.

\subsection{Method I}

In the first method (Method I ), we estimate the diffusion coefficient by comparing experimental time evolution of diffusion range (Fig. 3) with theoretical one. Diffusion time dependence of diffusion range can be calculated from quencher depth profiles based on Fick's law for various diffusion time (Fig. 5a), assuming that the film is soluble when quencher concentration (Q) is larger than a certain threshold. To examine this threshold, dissolution characteristics was examined for films containing various amount of quencher (Fig. 6). The same irradiation dose was used as for Fig. 3. The result shows that the film is soluble when quencher concentration is larger than 0.05. Assuming this, we obtain theoretical diffusion time dependence of diffusion range as shown in Fig. 5b. Comparing Fig. 3 with Fig. 5b, the diffusion coefficient of quencher DP was estimated to be $0.002 \mu \mathrm{m}^{2} / \mathrm{sec}$ at $100{ }^{\circ} \mathrm{C}$. 

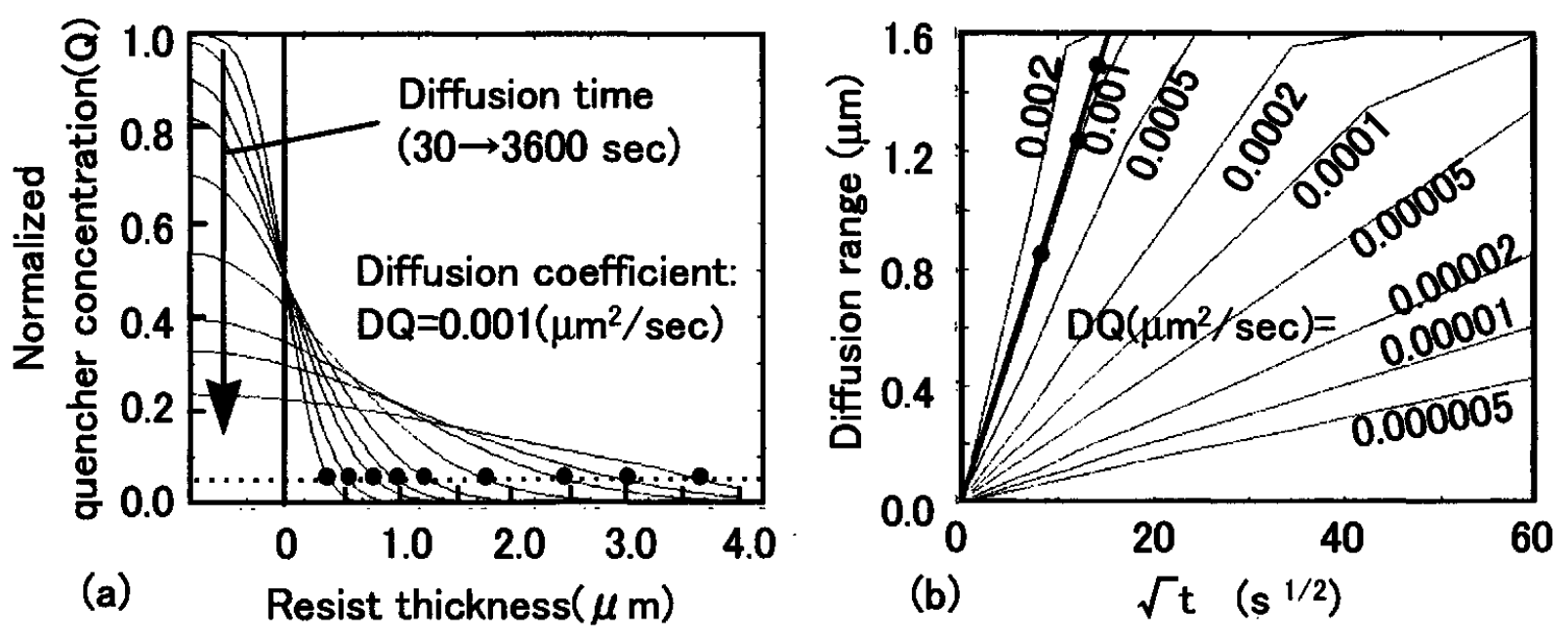

Fig. 5 Estimation method of quencher diffusion coefficient (Method I ).

(a) Calculated time evolution of quencher depth profile in resist.(DQ=0.001 $\mu \mathrm{m}^{2} / \mathrm{sec}$.)

(b) Time dependence of diffusion range. Diffusion range is defined by depth at $\mathrm{Q}=0.05$.

Diffusion coefficient for several quencher compounds was examined using the Method I . Results are summarized in Table. 1. The threshold concentration of 0.05 was assumed though this is not always clear for other quenchers. Estimated diffusion coefficients ranged between 0.0001 and $0.003 \mu \mathrm{m}^{2} / \mathrm{sec}$ at $100{ }^{\circ} \mathrm{C}$. These values correspond to diffusion length $(\sqrt{D t})$ of 0.09-0.52 $\mu \mathrm{m}$ for 90-sec PEB, which are considerably large compared with those reported for acid diffusion [3]. Temperature dependence of measured diffusion coefficient gives the activation energy of about $130 \mathrm{~kJ} / \mathrm{mol}$ (Fig. 7), which is close to those reported for acid diffusion in resists [6].

\subsection{Method II}

In Fig. 4, we measured the relationship between diffusion range and irradiation dose. Here, we assume that all the quencher in dissolved

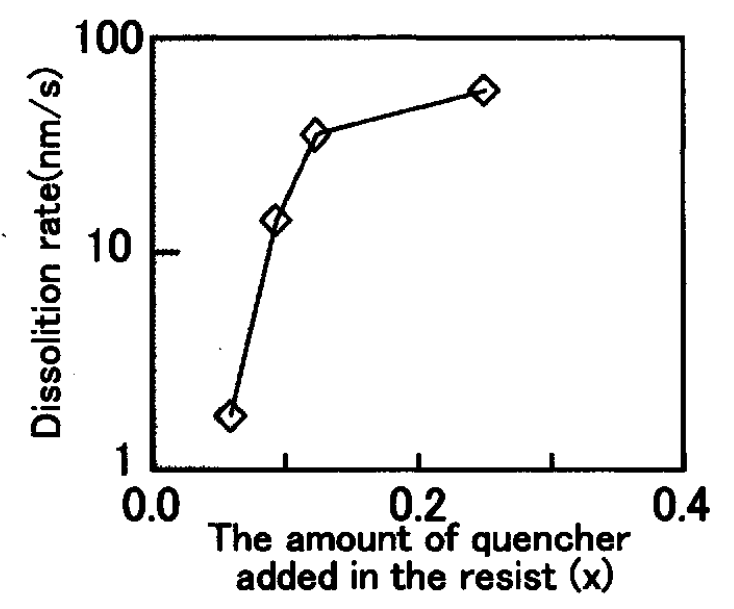

Fig. 6 Dissolution rate as a function of initial quencher concentration. Irradiation dose $=90 \mathrm{~mJ} / \mathrm{cm}^{2}$. Novolak(100)/triol-3(20)/DPIOTf(2.15) /2,2'-Dipyridyl $(0.78 \cdot x)($ wt ratio).

Table I Diffusion coefficient for several quenchers at various diffusion temperature. (measured by Method I )

\begin{tabular}{|c|c|c|c|c|c|}
\hline \multirow[b]{2}{*}{ Quencher } & & \multicolumn{4}{|c|}{ Diffusion temperature $\left({ }^{\circ} \mathrm{C}\right)$} \\
\hline & & 70 & 80 & 90 & 100 \\
\hline 2-Benzyl pyridine & (BP) & 0.000010 & 0.00007 & 0.0010 & 0.003 \\
\hline 1,3-di(4-pyridyl) propane & (DPP) & 0.000009 & 0.00007 & 0.0010 & 0.003 \\
\hline 2,2'-Dipyridyl & (DP) & 0.000007 & 0.00004 & 0.0007 & 0.002 \\
\hline 4,4'-Diphenyl-2,2'-dipyridyl & (DPhDP) & & 0.00001 & 0.0001 & 0.0007 \\
\hline 4-methyl aminopyridine & (AP) & & 0.000009 & 0.00005 & 0.0001 \\
\hline
\end{tabular}




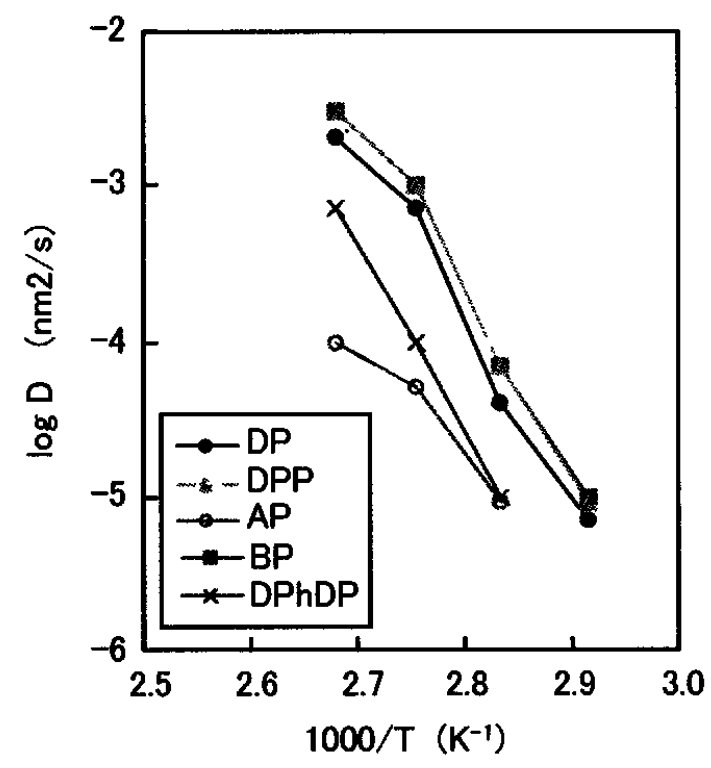

Fig. 7 Arrhenius plot of diffusion coefficient for several quenchers.

region of the film is consumed in quenching reaction, and a certain amount of acid $\Delta$ is necessary for changing the film insoluble. Because the amount of acid consumed in quenching reaction equals to that of quencher consumed, the quencher concentration $Q$ at the dissolution end point (bottom of hole) is smaller than initial acid concentration $H$ by $\Delta$ before quenching reaction. Thus, we can estimate the quencher concentration distribution as shown in Fig. 8, when diffusion ranges $\mathrm{d} 1-\mathrm{d} 4$ were observed for initial acid concentration H1-H4. Diffusion coefficient is further estimated by comparing the measured quencher depth profile with the simulated one. (Method II)

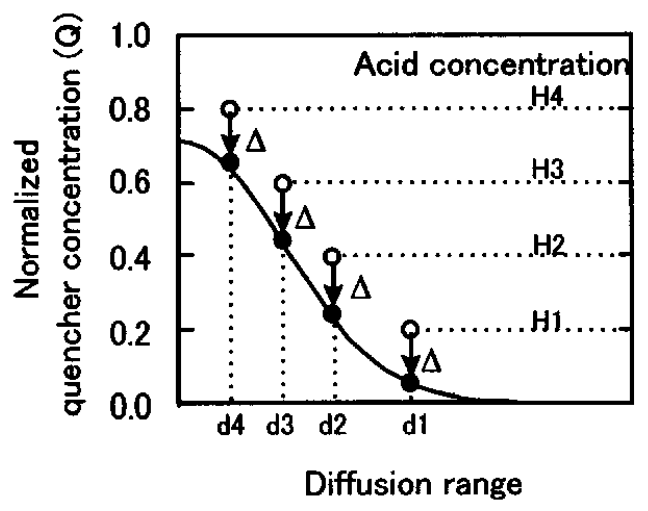

Fig. 8 Method of estimating quencher distribution (Method II).
To identify the amount of $\Delta$, the relationship between the amount of generated acid and the irradiation dose was examined (Fig. 9). Resist containing color indicator TBPB (Tetrabromo phenol Blue) was exposed at $i$-line and absorption change at $628 \mathrm{~nm}$ was measured. Measured absorption was converted to acid concentration using the calibration curve obtained from samples containing known amount of acid in the resist. Result showed that about 0.25 acid is generated at irradiation dose of $90 \mathrm{~mJ} / \mathrm{cm}^{2}$. Because the film exposed with the same irradiation dose changed soluble when the quencher concentration is larger than 0.05 (Fig. 6), we conclude that $\Delta$ is 0.2 .

Additionally, the sensitivity curve also plotted in Fig. 9 shows that about 0.2 acid is necessary for making the detector film insoluble, suggesting the effectiveness of above estimation.

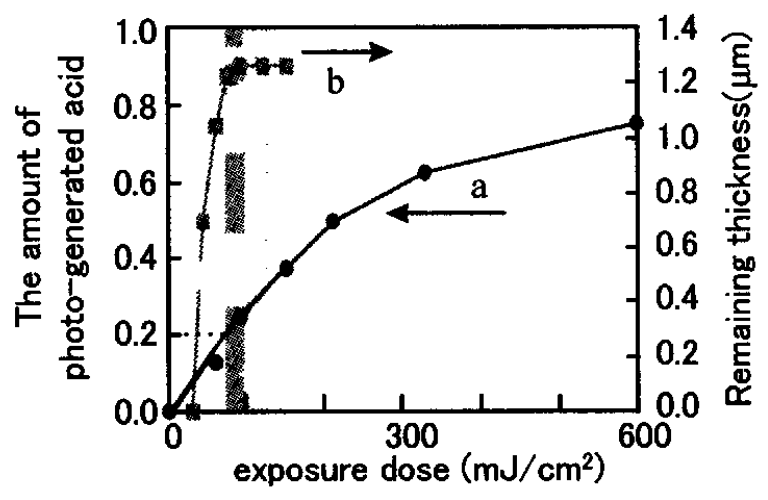

Fig. 9 Exposure dose dependence of the amount of photo-generated acid (a) and remaining resist thickness after development (b).

Composition for (a) is Novolak (100)/triol-3(20)/ DPIOTf (2.15) and added TBPB(2) for (b) (wt ratio) .

From Fig. 9, the initial acid concentration for the each film used in Fig. 4. (irradiated with 75 , 165,315 , and $630 \mathrm{~mJ} / \mathrm{cm}^{2}$ ) is found to be $0.2,0.4$, 0.6 and 0.8 . Thus the quencher concentration at the dissolution end point in each film is estimated approximately to be $0,0.2,0.4$, and 0.6 , respectively. Plotting the dissolution depth with respect to the quencher concentration estimated above for each film, we obtain the depth profile of quencher diffused into the detector film as shown in Fig. 10.

Comparing Fig. 10 with calculated results, the diffusion coefficient of 2,2'-Dipyridyl in the resist 
film is estimated to be about $0.002 \mu \mathrm{m}^{2} / \mathrm{sec}$ at $100{ }^{\circ} \mathrm{C}$. The results obtained from the two methods showed considerably good agreement.

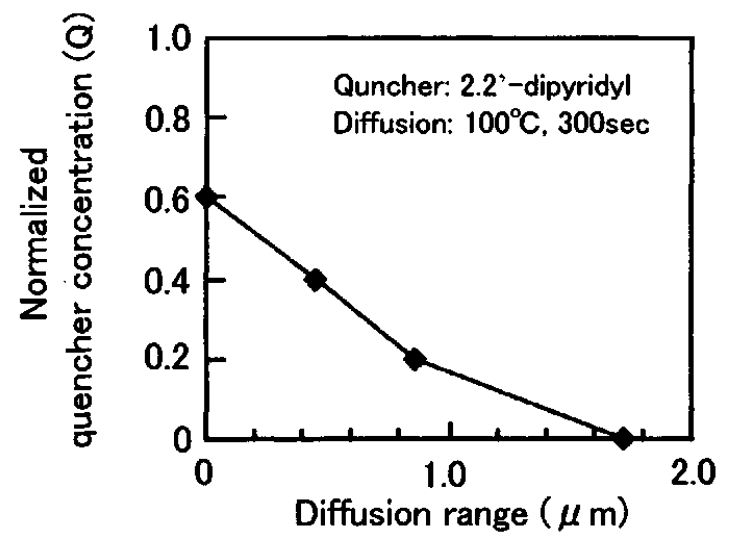

Fig. 10 Quencher depth profile obtained by Method II .

\section{Modeling of acid/quencher mutual diffusion}

To investigate the influences of quencher diffusion on resist patterning, we built a new kinetic model for chemically amplified resist. The photo-generated acid and quencher mutually diffuse in resist film and the acid-quenching reaction takes place. This process is formulated as follows.

$$
\begin{aligned}
& \partial \mathrm{H} / \partial \mathrm{t}=\nabla\left(\mathrm{D}_{\mathrm{H}} \nabla \mathrm{H}\right)-\mathrm{k} \cdot \mathrm{H} \cdot \mathrm{Q} \\
& \partial \mathrm{Q} / \partial \mathrm{t}=\nabla\left(\mathrm{D}_{\mathrm{Q}} \nabla \mathrm{Q}\right)-\mathrm{k} \cdot \mathrm{Q} \cdot \mathrm{H}, \\
& \mathrm{H}(\mathrm{t}=\mathbf{0})=\mathrm{I}, \quad \mathrm{Q}(\mathrm{t}=\mathbf{0})=\mathrm{R}
\end{aligned}
$$

where $H$ and $Q$ are acid and quencher concentration, $D_{H}$ and $D_{Q}$ are diffusion coefficients for acid and quencher, and $k$ is reaction coefficient for acid quenching reaction. The initial acid distribution is assumed to equal to the image intensity distribution (I), because actual wafer exposure is usually performed at the linear region in Fig. 9. The initial quencher distribution is assumed to be uniform (R).

In implementing the above model into our simulation software, it is assumed that quenching reaction is fast enough compared with the diffusion process. (The validity of this assumption is supported by the fact that the change in TBPB absorption saturated immediately after photo acid generation.) Then, the calculation process was simplified as illustrated in Fig. 11. Both $\mathrm{H}$ and Q are normalized by the concentration of acid generated by nominal exposure dose for a wide clear region on a mask.

\section{Simulation and exposure experiment}

\subsection{Procedures}

The model shown in the previous section was implemented into our in-house two-dimensional (planer) lithography simulator [7], and simulated results were compared with measured resist line widths on real wafers. Three parameters, acid diffusion length $(\mathrm{dH})$, quencher diffusion length $(\mathrm{dQ})$, and initial quencher concentration $\mathrm{R}$ were optimized to fit simulated results with measured wafer CDs best. The wafer experiments were performed with a $\mathrm{KrF}$ scanner $(\mathrm{NA}=0.68)$, darkfield-attenuated phase-shifting masks $(6 \%$ transmission) and a commercial positive-tone chemical amplification resist (acetal-base). Normal circular illumination $(\sigma=0.75)$ and annular illumination ( $\sigma=0.75-0.50$ ) were used. Simulations based on the pure optical model and the acid-diffusion (diffused aerial image) model

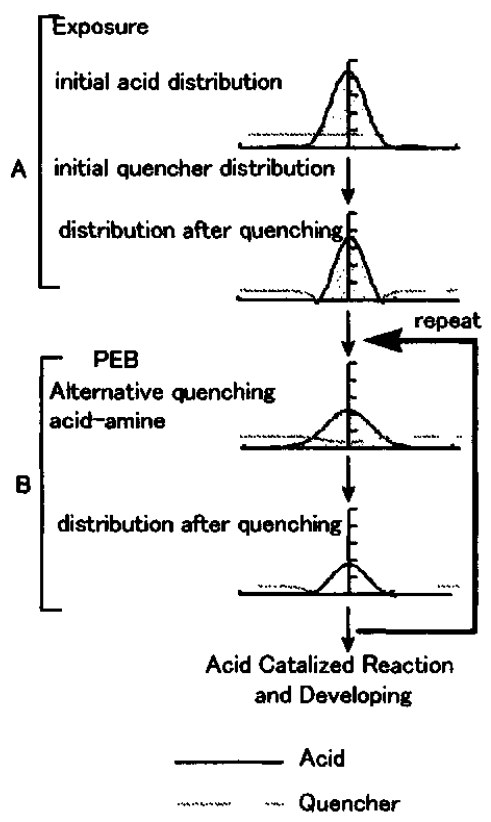

Fig. 11 Calculation procedure.

were also examined for comparison.

\subsection{1-D proximity effect}

One-dimensional (1-D) proximity effects were examined to test the effectiveness of the three models, optical, acid-diffusion, and acid-quencher mutual diffusion. Measured and simulated line widths are shown in Fig. 12a, b, and c as functions of mask pattern feature size (mask bias) for $0.4-\mu \mathrm{m}$ pitch 

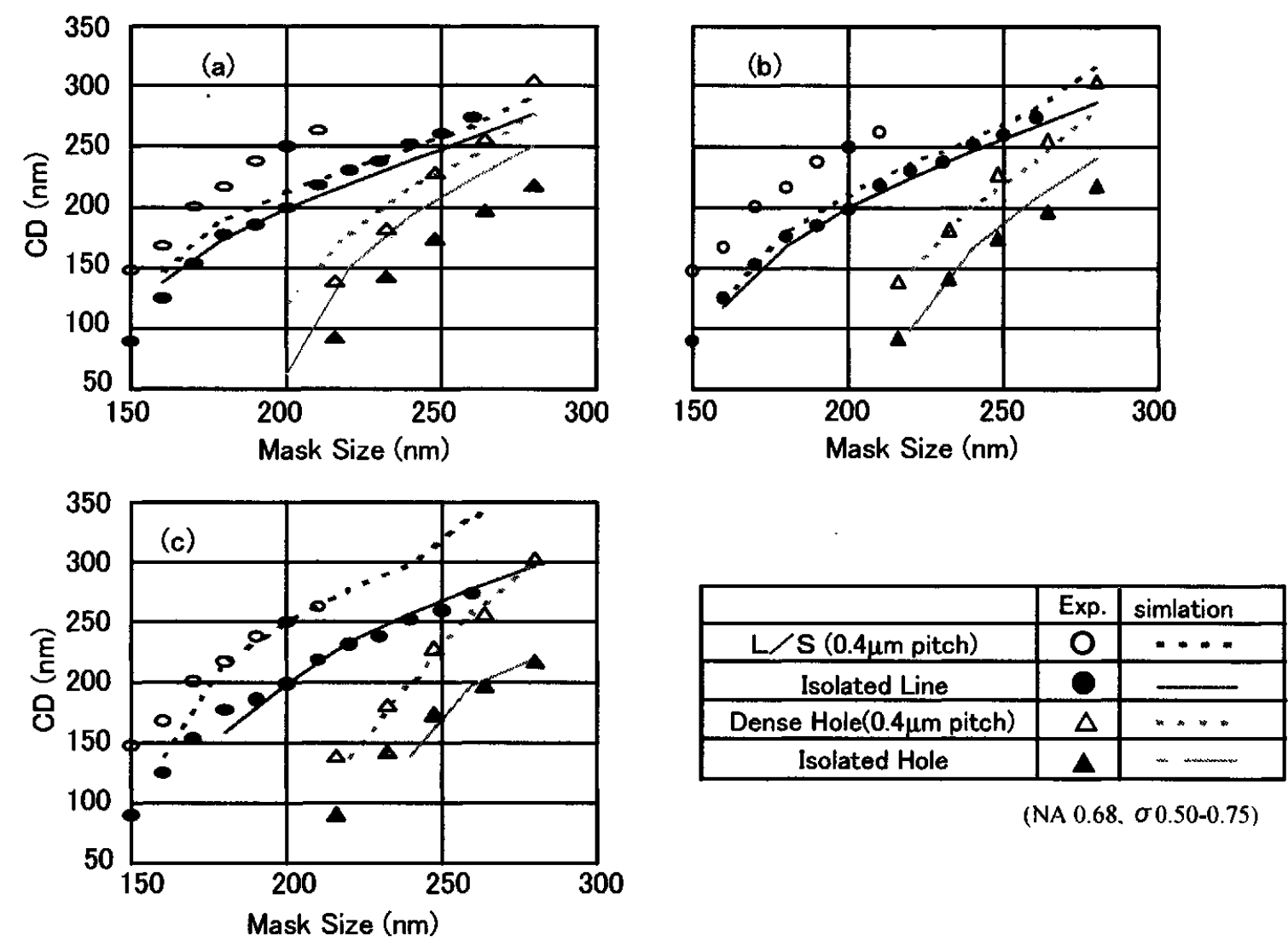

(NA 0.68, $\sigma 0.50-0.75$ )

Fig. 12 Printed feature size as functions of mask feature size.

(a) pure optical model, (b) acid diffusion model, (c) acid/quencher mutual diffusion model.

dense lines, isolated line (clear region on mask), 0.4- $\mu \mathrm{m}$ pitch dense windows, and isolated window. All experimental data were taken under the same exposure condition.

Measured line width showed a large pattern density dependence (also called iso-dense bias), and also printed feature size is significantly smaller for window-type patterns compared with line-type patterns. For example, wafer $\mathrm{CD}$ for $0.2-\mu \mathrm{m} \quad \mathrm{L} / \mathrm{S}$ approaches $0.25 \mu \mathrm{m}$ under the condition $0.2-\mu \mathrm{m}$ isolated line delineated as designed size. Either the pure optical model or the acid diffusion model is difficult to explain these behaviors (Fig. 12a and 12b, respectively). On the other hand, in our new model, both the isodense bias and the hole-line bias were successfully reproduced (Fig. 12c), assuming quencher diffusion length of $0.2 \mu \mathrm{m}$, acid diffusion length of $0.02 \mu \mathrm{m}$, and initial quencher concentration of 0.2 (normalized by maximum acid concentration in wide exposed region). The quencher diffusion length that gives the best result is much larger than for acid diffusion length. This agrees with the results from direct diffusion coefficient measurement discussed in the earlier section.

\subsection{Line end shortening}

Line-end shortening was examined as twodimensional proximity effects. On real wafers, the amount of line-end shortening strongly depends on the feature size of pattern facing to the line-end. Three cases, A, B, and C shown in Fig. 13a were tested, in each of which different size feature is facing to the line-end. Line-end shortening for the three cases were simulated with varying acid diffusion length (Fig. 13a). The experimental results are also shown in this figure. Though the line-end shortening increases with acid diffusion length, its dependence on the feature size of facing pattern is not reproduced. Similar calculation was carried out with varying quencher diffusion length (Fig. 13b). For the quencher diffusion length larger than $0.1 \mu \mathrm{m}$, the simulated line-end shortening approaches to the 


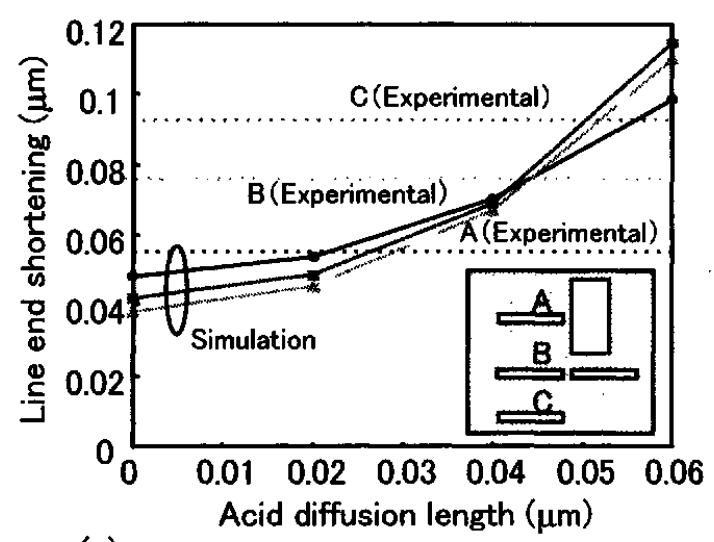

(a)

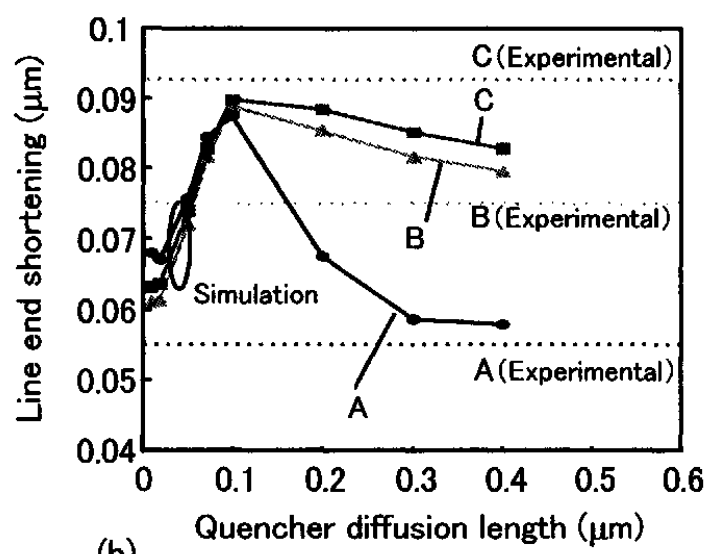

(b)

Fig. 13 Line end shortening as a function of acid /quencher diffusion length.

(a) acid diffusion model, (b) acid/quencher mutual diffusion model $(\mathrm{dH}=0.04, \mathrm{R}=0.20)$.

experimental results, suggesting the influence of quencher diffusion on line-end shortening behavior.

\section{Conclusions}

Diffusion of quencher contained in chemically amplified resists was discussed. Measured diffusion lengths of typical quencher compounds were significantly large compared with those reported for acid. A new resist model including acid/quencher mutual diffusion and quenching reaction was introduced and implemented into our in-house lithography simulator. Simulated results based on the new model explained large proximity effects observed on real wafers, which were difficult to be explained with other models such as the pure optical model and diffused aerial image model.

In conclusion, quencher diffusion can have significant influences on macroscopic behavior of resist materials such proximity effects. The model is applicable to fast pattern geometry simulation, which can be a base for high accuracy OPC verification and OPC rule generation. Also, we expect that better understanding of relation between material composition and imaging characteristics is useful for controlling macroscopic behavior of resist materials.

\section{References}

1. S. Saito, N. Kihara, T. Naito, M. Nakase, T. Nakasugi, and Y. Kato, Proc. SPIE., 3049, 658 (1997).

2. W. Huang, Proc. SPIE., 3678, 1040 (1999).

3. L. Schlegel, T. Ueno, N. Hayashi, and T. Iwayanagi, Jpn. J. Appl. Phys., 30, 3132 (1991).

4. S. Uchino, J. Yamamoto, S. Migitaka, K. Kojima, M. Hashimoto, F. Murai, and $H$. Shiraishi, J. Photopolym Sci. Technol., 11, 555 (1998).

5. S. Migitaka, J. Yamamoto, S. Uchino, M. Hashimoto, F. Murai, and H. Shiraishi, Jpn. J. Appl. Phys., 30, 3132 (1991).

6. K. Asakawa, J. Photopolym Sci. Technol., $\underline{6}$, 505 (1993).

7. H. Fukuda and K. Hattori, Proc. SPIE., 3334, 154 (1998). 